\title{
MMM-CADIAG: A MINI MODIFIED CADIAG WITH MOBIUS TRANSFORM
}

\author{
NGUYEN HOANG PHUONG ${ }^{(1)}$
}

\begin{abstract}
Abatract . This paper describes the MMM-CADIAG system called Mini Modifid CADIAG with Moebius transform which is based on the algorithm of Mobius transform for CADIAG-2. This algorithm using Mobius trasform to compute new rule base for CADIAG-2. To apply Mobius transform for CADIAG-2 means to find new weights of fuzzy rules. This algorithm guarantees that using generalized MaxMin inference of CADIAG-2 the inference machine will reproduce the expert's stated conditional beliefs as total degrees of confirmation and exclusion. MMM-CADIAG uses positive and negative knowledge. Knowledge Base of the system consists of set of IF - THEN rules. Each rule assigns its weight in $[0,1]$. With assumption that relative frequencies are used as weights of rules. MMM-CADIAG is able to calculate new weights of fuzzy rules and then suggest diagnoses by using the Mini Modified inference engine of CADIAG-2. Finally, a computer test program with established simple knowledge base in Oriental Medicine as an example is developed and tested. Programs is developed in C++ programming language and can run on PC/IBM computers.
\end{abstract}

\section{INTRODUCTION:}

CADIAG-2 is a Medical Fuzzy Expert System for Diagnosis which have been developed and applied successfuly in many medical applications as in Rheumatology, Pancrea, GallBaldder etc. This system is as the physician's assistent in clinical diagnosis and also as an instructual system devoted to medical education [1], [9], [10], [11], [12]. Knowledge base of CADIAG-2 consists also of relationships between symptoms, sysmptom - combinations and diseases. These relationships are represented in the form of rule IF (antecedent) THEN (consequent) with two parameters: o- frequency of occurrence of symptom (or symptom combination) with disease and $\mathrm{c}$ - confirmation degree of symptom (or symptom - combination) for disease. The aim of our project is to analyze, improve and generalize CADIAG-2: a diagnostic expert system using fuzzy logic and fuzzy set theory for internal medicine by including negative knowledge, by combining negative and positive evidence and modifying support scores of CADIAG-2 [5], [14] and to study some relations between CADIAG-2 and MYCIN-like systems [2], [15], and also to elaborate a Mobius transform for CADIAG-2 [4], [16]. This algorithm guarantees that using generalized MaxMin inference of CADIAG-2 the inference machine will reproduce the expert's stated conditional beliefs as total degrees of confirmation and exclusion. This pape: describes the MMM-CADIAG system called Mini Modified CADIAG with Mobius traisform. Knowledge of the system consists of negative and positive evidences. Knowledge base of the system is represented in the form of IF - THEN rules. With assumption that relative frequencies are used as weight of rules. First, the Mobius transform for CADIAG-2 is applied to calculate new weight of the rules, then the inference engine of MMM-CADIAG bnsed on MaxMin conposition of rules combining negative and positive knowledge will infer diagnoses. The aim of this work is the design and experimental implementation of MMM-CADIAG a Mini Modified CADIAG with Mobius transform. This system is an generarized version of CADIAG-2 by making some mini modification of CADIAG-2 with assumption that degrees of truth of rules in MMM-CADIAG are used as relative frequencies or their fuzzifications. Some properties of the system are as follows:

- Extending fuzzy negative knowledge to MMM-CADIAG. 
- Combining negative and positive knowledge to infer diagnoses as well as 'confirmed', 'disconfirmed', 'likely', 'unlikely', 'unknown' diagnoses.

- At first, MMM-CADIAG applies Mobius transform algorithm for CADIAG-2 for finding new weights of fuzzy rules such that using generalized MaxMin inference of CADIAG-2, the inference of MMM-CADIAG will reproduce the expert's stated conditional beliefs as total degrees of confirmation and exclusion.

The paper is organized as follows: Section 2 presents some notions of Mobius transform for CADIAG-2. Section 3 describes a structure of MMM-CADIAG. Section 4 shows the implementation of the prototype of MMM-CADIAG. Finally, the conclusion is given.

\section{MOBIUS TRANSFORM FOR CADIAG-2}

Let us recall some main difinitions of the Mobius transform algorithm for CADIAG-2 defined in [4] for understanding of the nature of components of MMM-CADIAG will described bellow.

Definition 1: A fuzzy patient data of patient $\mathrm{P}_{\mathrm{q}}$ consists of values $\mu_{\mathrm{R}_{\mathrm{PS}}}^{+}\left(\mathrm{P}_{\mathrm{q}}, \mathrm{S}_{\mathrm{i}}\right)$ - degree of confirmation and $\mu_{R_{p s}}^{-}\left(P_{q}, S_{i}\right)$ - degree of exclusion for $i=1, \ldots ., m$. Assume that, at least, $\mu_{\mathrm{R}_{\mathrm{PS}}}^{+}\left(\mathrm{P}_{\mathrm{q}}, \mathrm{S}_{\mathrm{i}}\right)$ or $\mu_{\mathrm{R}_{\mathrm{ps}}}^{-}\left(\mathrm{P}_{\mathrm{q}}, \mathrm{S}_{\mathrm{i}}\right)=0$.

Definition 2: The patient data $\mu_{\mathrm{R}_{\mathrm{PS}}}^{+}\left(\mathrm{P}_{\mathrm{q}}, \mathrm{S}_{\mathrm{i}}\right), \mu_{\mathrm{R}_{\mathrm{ps}}}^{-}\left(\mathrm{P}_{\mathrm{q}}, \mathrm{S}_{\mathrm{i}}\right)$ (for $\left.\mathrm{i}=1, \ldots, \mathrm{m}\right)$ are three-valued for patient $\mathrm{P}_{\mathrm{q}}$, if for all $\mathrm{S}_{\mathrm{i}}, \mu_{\mathrm{R}_{\mathrm{PS}}}^{+}\left(\mathrm{P}_{\mathrm{q}}, \mathrm{S}_{\mathrm{i}}\right)$ and $\mu_{\mathrm{R}_{\mathrm{PS}}}^{-}\left(\mathrm{P}_{\mathrm{q}}, \mathrm{S}_{\mathrm{i}}\right)$ take value 0 or 1 .

- $\mu_{R_{P S}}^{+}\left(P_{q}, S_{i}\right)=0$ and $\mu_{R_{P S}}^{-}\left(P_{q}, S_{i}\right)=0$ mean symptom $S_{i}$ - unknown for patient $P_{4}$

- $\mu_{R_{P S}}^{+}\left(P_{q}, S_{i}\right)=1$ means symptom $S_{i}$ - surely present for patient $P_{4}$.

- $\mu_{R_{P s}}^{-}\left(P_{4}, S_{i}\right)=1$ means symptom $S_{i}$ - surely absent for patient $P_{4}$.

The $\mu_{R_{p s}}^{+}\left(P_{q}, S_{i}\right)$ and $\mu_{R_{p s}}^{-}\left(P_{q}, S_{i}\right)$ determine an elementary conjunction $E_{k}$ of symptoms $S_{i}$ such that $S_{i}$ occurs in $E_{k}$ possitively if $\mu_{R_{P S}}^{+}\left(P_{4}, S_{i}\right)=1$ and negatively if $\mu_{R_{P S}}^{-}\left(P_{4}, S_{i}\right)=1$.

Definition 3: An elementary conjunction $E_{k}$ of symptoms $S_{i}$ is defined by

(recall the notion $(0) \mathrm{S}_{\mathrm{i}}=>\mathrm{S}_{\mathrm{i}}$, (1) $\mathrm{S}_{\mathrm{i}}=\mathrm{S}_{\mathrm{i}}$ )

$$
\mathrm{E}_{\mathrm{k}}=\left(\varepsilon_{1}\right) \mathrm{S}_{1} \& \ldots . \&\left(\varepsilon_{\mathrm{m}}\right) \mathrm{S}_{\mathrm{m}}
$$

Knowing $\mu_{R_{P S}}^{+}\left(P_{q}, S_{i}\right), \mu_{R_{P S}}^{-}\left(P_{q}, S_{i}\right)$ we define $\mu_{R_{P S}}^{+}\left(P_{q}, E_{k}\right)$ in the obvious way.

Knowing relation $R_{S D}^{+}$for confirmation of diagnosis (see details in [1]), we extend CADIAG-2 by a relation $R_{S D}^{-}$defined by $\mu_{R_{P D}}\left(E_{k}, D_{j}\right)\left(E_{k}\right.$ is a symptom or elementary conjunction of symptom) in $[0,1]$, where the value $\mu_{R_{p D}}\left(E_{k}, D_{j}\right)$ indicates degree in which a symptom (or elementary conjunction of sumptoms) $\mathrm{E}_{\mathrm{k}}$ exclued a diagnosis $\mathrm{D}_{\mathrm{j}}$. Thus, the following MaxMin composition of rules proposed and used to deduce the degrees of confirmation and exclusion of the disease $D_{j}$ for the patient $P_{q}$ from the observed symptoms $E_{k}$ are follows: 


$$
\mathrm{R}_{\mathrm{SD}}^{+}=\mathrm{R}_{\mathrm{PS}} \mathrm{O} \mathrm{R}_{\mathrm{SD}}^{-}
$$

defined by:

$$
\begin{gathered}
\mu_{R_{P D}^{+}}\left(P_{q}, D_{j}\right)=\operatorname{Max}_{E_{k} \in S y s} \operatorname{Min}\left(\mu_{R_{P S}}^{+}\left(P_{q}, E_{k}\right) ; \mu_{R_{S p}^{+}}\left(E_{k}, D_{j}\right)\right. \\
R_{P D}^{-}=R_{P S} O R_{P D}^{-}
\end{gathered}
$$

defined by

$$
\mu_{R_{\text {PD }}^{-}}\left(\mathrm{P}_{\mathrm{q}}, \mathrm{D}_{\mathrm{j}}\right)=\operatorname{Max}_{\mathrm{E}_{\mathrm{k}} \in S y s} \operatorname{Min}\left(\mu_{\mathrm{R}_{\mathrm{PS}}}^{+}\left(\mathrm{P}_{\mathrm{q}}, \mathrm{E}_{\mathrm{k}}\right) ; \mu_{R_{S D}^{-}}\left(\mathrm{E}_{\mathrm{k}}, \mathrm{D}_{\mathrm{j}}\right)\right.
$$

where Sys - a set of symptoms $\mathrm{E}_{\mathrm{k}}$.

Remark: Note that the patient data are three-value, i. e. given by an elementary conjucntion $E_{k}$, then this reduces to $\mu_{R_{p d}^{+}}\left(P_{q}, D_{j}\right)=\operatorname{Max}_{E_{k} \subseteq E_{k}}\left(\mu_{R_{p D}^{+}}\left(E_{k}^{\prime}, E_{j}\right)\right)$ and it is similar for $\mu_{R_{P D}}\left(P_{q}, D_{j}\right)$.

Let us recall some notions on group operation $\Theta$ and $\Theta$ on $(-1,1)$ in [3], [7] which will be used later.

- The PROSPECTOR group operation $\oplus$ on $(-1,1)$ is defined as follows:

$$
x \oplus y=\frac{x+y}{1+x y}
$$

- Operation $\Theta$ is a group operation defined $\mathrm{x} \Theta \mathrm{y}=\mathrm{x} \oplus-\mathrm{y}$

Definition 4: A rule base $\Theta$ given by $\mu_{R_{S D}^{+}}\left(\mathrm{E}_{\mathrm{k}}, \mathrm{D}_{\mathrm{j}}\right)$ and $\mu_{R_{S D}^{-}}\left(\mathrm{E}_{\mathrm{k}}, \mathrm{D}_{\mathrm{j}}\right)$ consists of rules:

$$
\left.\mathrm{E}_{\mathrm{k}} \rightarrow \mathrm{D}_{\mathrm{j}}\left(\mu_{R_{S D}^{ \pm}}\left(\mathrm{E}_{\mathrm{k}}, \mathrm{D}_{\mathrm{j}}\right)\right), \mathrm{E}_{\mathrm{k}} \rightarrow \mathrm{D}_{\mathrm{k}}\left(\mu_{R_{S D}^{-\bar{D}}}\left(\mathrm{E}_{\mathrm{k}}, \mathrm{D}_{\mathrm{j}}\right)\right)\right)
$$

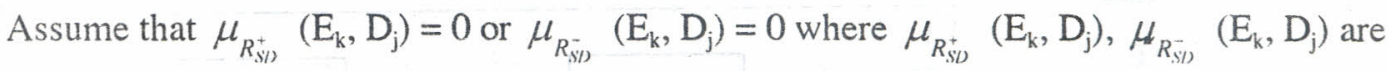
weights of fuzzy rules in $[0,1]$.

Definition 5: Given a patient data, the total degree for confirmation and exclusion of diagnosis $D j$ by patient $P_{4}$ from ot served symptom $S_{i}$ is:

$$
\mu_{R_{P Q}^{\text {pop }}}\left(P_{q}, D_{j}\right)=\mu_{R_{p p}^{+}}\left(P_{q}, D_{j}\right) \Theta \mu_{R_{p p}^{-}}\left(P_{q}, D_{j}\right)
$$

in $[-1,1]$.

Definition 6: A conditional weight system $\beta$ consists of $\beta_{S D}^{+}\left(D_{j} \mid E_{k}\right)$ and $\beta_{S D}^{-}\left(D_{j} / E_{k}\right)$ in $[0$, 1] for a set of pairs $\left(D_{j}, E_{k}\right)$. Assunie that $\beta_{S D}^{+}\left(D_{j} \mid E_{k}\right)=0$ or $\beta_{S D}^{-}\left(D_{j} \mid E_{k}\right)=0$, where $E_{k}$ : an elementary conjunction of symptoms $\mathrm{S}_{\mathrm{i}}$.

Definition 7: A total conditional weight system $\beta_{S D}^{\text {tot }}\left(D_{j} \mid E_{k}\right)$ for a set of pairs $D_{j} \in$ Dise (Dise: a set of Diseases $D_{j}$ ), $E_{k} \in E C$ (Sym) (Elementary Conjunction of Symptoms) isdefined as follows:

$$
\beta_{S D}^{\text {tot }}\left(D_{j} \mid E_{k}\right)=\beta_{S D}^{+}\left(D_{j} \mid E_{k}\right) \bullet \beta_{S D}^{-}\left(D_{j} \mid E_{k}\right)
$$

Definition 8: A conditional weight system $\beta$ is weakly sound if the following holds for each $E_{k}^{\prime} \subseteq E_{k} \in E C(\operatorname{Sym})$ and $D_{j} \in$ Dise: if $\beta_{S D}^{+}\left(D_{j} \mid E_{k}\right), \beta_{S D}^{-}\left(D_{j} \mid E_{k}\right), \beta_{S D}^{+}\left(D_{j} \mid E_{k}^{\prime}\right)$, 
$\beta_{\mathrm{SD}}^{-}\left(\mathrm{D}_{\mathrm{j}} \mid \mathrm{E}_{\mathrm{k}}^{\prime}\right)$ are defined and $\beta_{\mathrm{n}}^{+}\left(\mathrm{D}_{\mathrm{j}} \mid \mathrm{F}^{\prime} \quad \beta_{\mathrm{SD}}^{-}\left(\mathrm{D}_{\mathrm{j}}, \mathrm{L}_{\mathrm{k}}\right)\right.$ is extremal (i.e = 1) (one of them takes value 0 ), then

$$
\left.\beta_{S D}^{+}\left(D_{j} \mid E_{k}^{\prime}\right)=\beta_{S D}\left(D \mid E_{k}\right), \beta_{S D}^{-}\left(D_{j} \mid E_{k}^{\prime}\right)=\beta_{S D}^{-}\left(D_{j} \mid E_{k}\right), 1\right)
$$

Theorem:

Let $\beta$ be a weakly sound conditional weight system. The: there is a rule base $\Theta$ weights $\mu_{R_{\mathrm{pD}}^{+}}\left(\mathrm{S}_{\mathrm{i}}, \mathrm{D}_{\mathrm{j}}\right)$ and $\mu_{R_{s / 2}}\left(\mathrm{~S}_{\mathrm{i}}, \mathrm{D}_{\mathrm{j}}\right)$ of fuzzy rules such that for each patient $\mathrm{P}_{\mathrm{q}}$ and each three-valued patient data $\mu_{R_{p s}}^{+}\left(P_{4}, S_{i}\right) ; \mu_{R_{p s}}\left(P_{4}, S_{i}\right)$ (therefore $E_{k}$ exists)

$$
\mu_{R_{P D}^{\text {rot }}}\left(P_{y}, D_{j}\right)=\beta_{S D}^{\text {tot }}\left(D_{j} \mid E_{k}\right)(5)
$$

whenever the right hand side is defined.

The weights of fuzzy rules are calculated based on the algorithm described in [4].

\section{STRUCTURE OF MMM-CADIAG}

MMM-CADIAG consists of five main conmonents: Conditional Weight System Module (CWSM), Mobius Transform Module (MTM) Mule Base Module (CRB), VIEW Module (VIEWM) and Inference Engine Module (IEM). The structure of MMM-CADIAG is described in Figure 1.

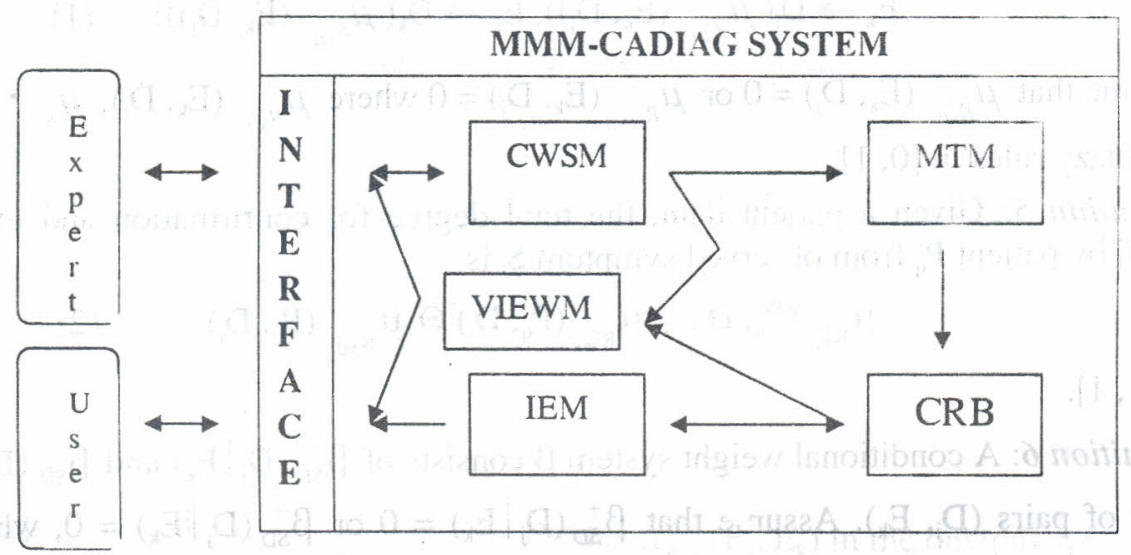

Figure 1: Structure of MMM-CADIAG

The main task MMM-CADIAG is to use M• bius transform to correct the weights of fuzzy rules and then to provide Diagnosis. The M* bius Transform task of MMM-CADIAG; consists of three subtasks: Entering Conditional Weight System (CWS), Viewing entered CWS, and Viewing New Rule Base. The tasks of MMM-CADIAG are shown in Figure 2. 


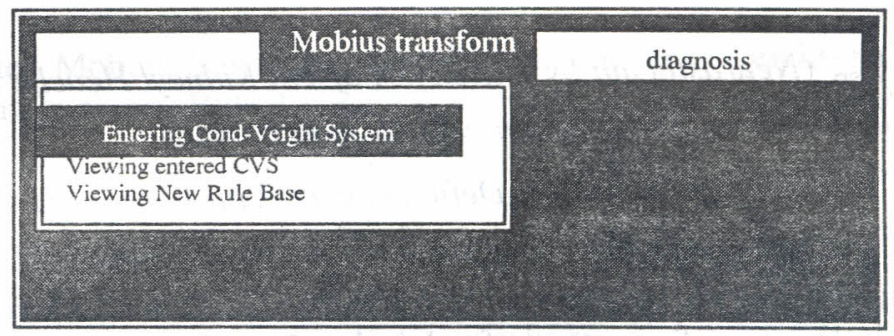

Figure 2: The Task of MMM-CADIAG

\subsection{Conditional Weight System Module (CWSM)}

Domain expert can use this module to enter conditional weight system $\beta$ into the system. Conditional weight system $\beta$ consits of some pairs $D_{j}$ (Diseases $D_{j}$ ), (elementary conjuction of Symptoms ${ }_{i}$ ), (for exampe, $\beta_{S D}^{+}\left(D_{j} \mid E_{q}\right), \beta_{S D}^{-}\left(D_{j} \mid E_{q}\right)$ ) and associating to each pair $(D, S)$ in the domain of $\beta$ a weight in $[0,1]$. Assume that $\beta_{S D}^{+}\left(D_{j} \mid E_{q}\right)=0$ or $\beta_{S D}^{-}\left(D_{j} \mid E_{q}\right)=0$.

A simple example of Conditional Weight System $\beta$ in Oriental Medicial is given as follow:

Rule 1:

$$
\begin{aligned}
& \beta_{\mathrm{SD}}^{+}(\text {Neurasthenia by Deficiency of Yin Kidney Headache })=0.4 \\
& \beta_{\mathrm{SD}}^{-}(\text {Neurasthenia by Deficiency of Yin Kidney• Headache })=0.0
\end{aligned}
$$

Rule 2:

$$
\begin{aligned}
& \beta_{\mathrm{SD}}^{+}(\text {Neurasthenia by Deficiency of Yin Kidney•Drowsiness })=0.3 \\
& \beta_{\mathrm{SD}}^{-}(\text {Neurasthenia by Deficiency of Yin Kidney• Drowsiness })=0.0
\end{aligned}
$$

Rule 3:

$\beta_{\mathrm{SD}}^{+}$(Neurasthenia by Deficiency of Yin Kidney• Tinghing in the ears) $=0.2$

$\beta_{\mathrm{SD}}^{-}$(Neurasthenia by Deficiency of Yin Kidneye Tinghing in the ears) $=0.0$

Rule 4:

$\beta_{\mathrm{SD}}^{+}($Neurasthenia by Deficiency of Yin Kidney Lumbar Pain $)=0.3$

$\beta_{\mathrm{SD}}^{-}$(Neurasthenia by Deficiency of Yin Kidney Lumbar Pain $)=0.0$

Rule 5:

$\beta_{\mathrm{SD}}^{+}($Neurasthenia by Deficiency of Yin Kidney•Pulse: tense, small $)=0.5$

$\beta_{\mathrm{SD}}^{-}$(Neurasthenia by Deficiency of Yin Kidney• Pulse: tense, small $)=0.0$

Rule 6:

$\beta_{\mathrm{SD}}^{+}($Neurasthenia by Deficiency of Yin Kidney• Bitter mouth $)=0.0$

$\beta_{\mathrm{SD}}^{-}($Neurasthenia by Deficiency of Yin Kidney Bitter mouth $)=0.7$

Rule 7: 
$\beta_{\mathrm{SD}}^{+}$(Neurasthenia by Deficiency of Yin Kidney/ Heache $\wedge$ Drowsiness $\wedge$ Tinghing in the ears $\wedge$ LumbarPain $\wedge$ Bitter mouth $)=0.4$

$\beta_{\mathrm{SD}}^{-}$(Neurasthenia by Deficiency of Yin Kidney/ Headache $\wedge$ Drowsiness $\wedge$ Tinghing in the ears $\wedge$ Lumbar Pain $\wedge$ Bitter mouth $)=0.0$

To simplify, we denote: Heat sands for Headache, Dro stands for Drowsiness, Tin stands for Tinghing in the ears, Lum stands for Lumbar Pain, Pul stands for Pulse: tense, small, Bit stands for Bitter mouth and Neur stands for Neurasthenia by Dificiency of Yin Kidney.

Example of Entered Weights by MMM-CADIAG is shown in Figure 3.

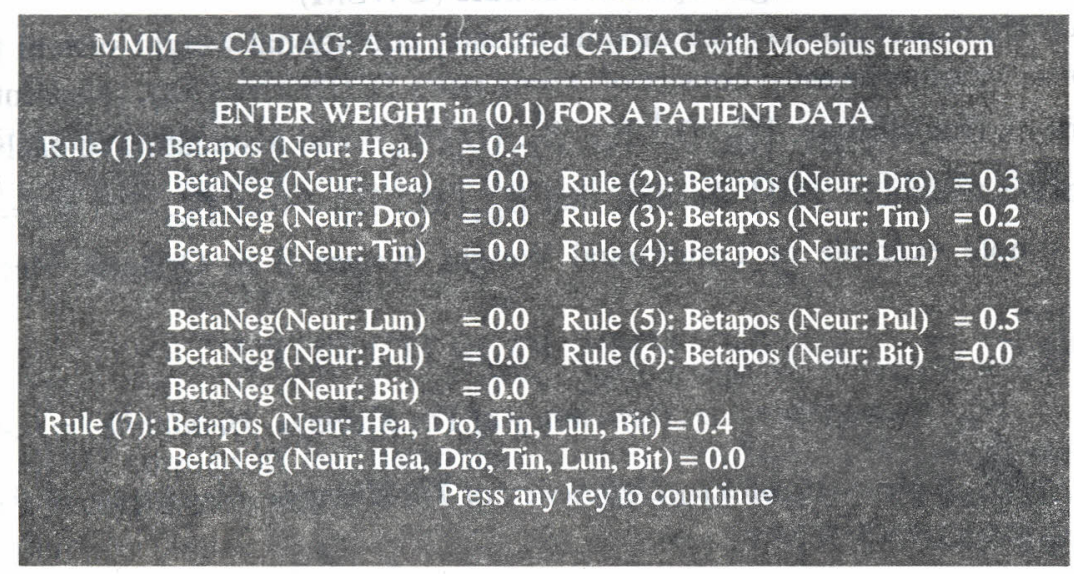

Figure 3: Enter Conditional Weight System

In our example, in Rule (1), when BetaPos(Neur - Hea, ) $=0.4$, then BetaNeg (Neur - Hea, ) receive automatically the value 0.0 , because $\beta_{S D}^{+}\left(D_{j} \bullet E_{q}\right)=0$ or $\beta_{S D}^{-}\left(D_{j} \bullet E_{q}\right)=0$. Weights can be acquired from several sources:

- From patient data base.

- From Medical Experts.

- From mixed sources (patient data base and Medical Experts)

The entered conditional weight system is stored in file: CWS.DAT in the following from:

1

Neur

0.4

0.0

1

Hea

where, 1 - rule number (integer), Neur - disease (string), 0.4 - value of $\beta^{+}$(Neur• Hea), 0.0 - value of $\beta^{-}$(Neur Hea), 1 - number (integer) of symptoms in the rule, Hea - sumptom (string).

Every item is stored in separate line. 
This module applies Mobius transform algorithm to fine new weights of fuzzy rules. Using MTM for the example above, Rule 1 - Rule 6 are satisfied to case 1 of algorithm in [4], then we get:

Rule 1:

$\mu_{\mathrm{R}_{\mathrm{SD}}^{+}}($Headache, Neurasthenia by Deficiency of Yin Kidney) $=0.4$

$\mu_{R_{S D}}$ (Headache, Neurasthenia by Deficiency of Yin Kidney) $=0.0$

Rule 2:

$\mu_{\mathrm{R}_{\mathrm{SD}}^{+}}$(Drowsiness, Neurasthenia by Deficiency of Yin Kidney) $=0.3$

$\mu_{R_{S D}}$ (Drowsiness, Neurasthenia by Deficiency of Yin Kidney) $=0.0$

Rule 3:

$\mu_{\mathrm{R}_{S D}^{+}}$(Tinghing in the ears, Neurasthenia by Deficiency of Yin Kidney) $=0.2$

$\mu_{\mathrm{R}_{S D}^{-}}$(Tinghing in the ears, Neurasthenia by Deficiency of Yin Kidney) $=0.0$

Rule 4:

$\mu_{\mathrm{R}_{\mathrm{SD}}^{+}}($Lumbar Pain, Neurasthenia by Deficiency of Yin Kidney) $=0.3$

$\mu_{\mathrm{R}_{\mathrm{SD}}}($ Lumbar Pain, Neurasthenia by Deficiency of Yin Kidney) $=0.0$

Rule 5:

$\mu_{R_{S D}^{+}}(P$ ulse: tense, small, Neurasthenia by Deficiency of Yin Kidney) $=0.5$

$\mu_{\mathrm{R}_{\mathrm{SD}}}$ (Plulse: tense, small, Neurasthenia by Deficiency of Yin Kidney) $=0.0$

Rule 6:

$\mu_{\mathrm{R}_{\mathrm{SD}}^{+}}$(Bitter mouth, Neurasthenia by Deficiency of Yin Kidney) $=0.0$

$\mu_{R_{S D}^{-}}$(Bitter mouth, Newia thenia by Deficiency of Yin Kidney) $=0.7$

Rule 7 is satisfied to case 3 (see in [4]), we have:

$$
\begin{aligned}
& M^{+}=\max (0.4,0.3,0.2,0.5,0.0)=0.5 \\
& M^{*}=\max (0.0,0.0,0.0,0.0 .0 .7)=0.7
\end{aligned}
$$

and

$$
M^{\text {tot }}=M^{+} \Theta M^{-}=0.5 \Theta 0.7=-0.416667
$$

On the other hand

$\beta_{\mathrm{SD}}^{\text {tot }}$ (Neurasthenia by Deficiency of Yin Kidney Headache $\wedge$ Drowsiness $\wedge$ Tinghing in the ears $\wedge$ Lumbar Pain $\wedge$ Bitter mouth $)=0.4-0.0=0.4<M^{\text {tot }}$.

Then

$\mu_{R_{S D}^{+}}$(Headache $\wedge$ Drowsiness $\wedge$ Tinghing in the ears $\wedge$ Lumbar Pain $\wedge$ Bitter mouth; Neurasthenia by Deficiency of Yin Kidney) $=0.859275$ and 
$\mu_{\left.R_{\mathrm{s}}\right)}$ (Headache $\wedge$ Drowsiness $\wedge$. Tinghing in the ears $\wedge$ Lumbar Pain $\wedge$ Bitter mouth; Netrasthenia by Deficiency of Yin Kidney) $=0.859275$ and

$\mu_{R_{i i}}$ (Headache $\wedge$ Drowsiness $\wedge$ Tinghing in the ears $\wedge$ Lumbar Pain $\wedge$ Bitter mouth; Neurasthenia by Deficiency of Yin Kidney) $=0.00000$

The Figure 4 is the result of calculation for new weights of the given example.

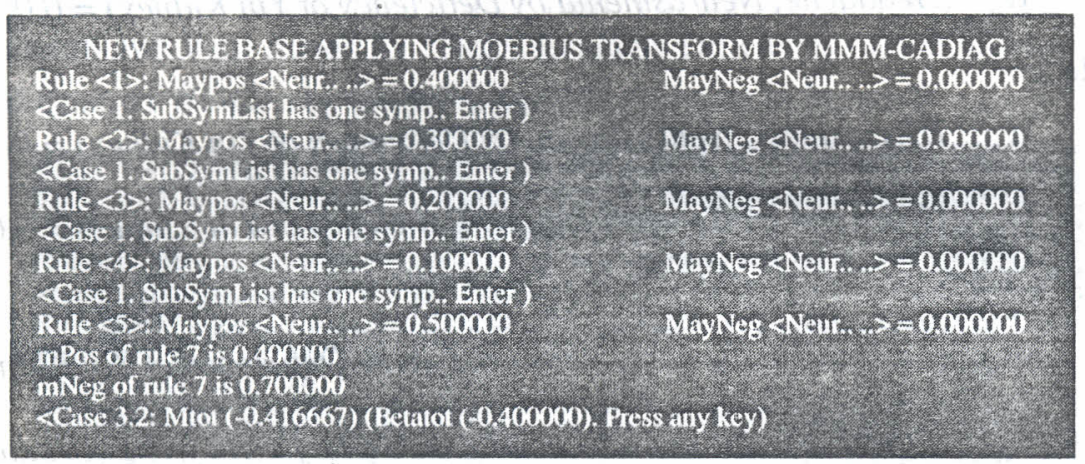

Figure 4: New weights of Rules

\subsection{Corrected Rule Base (CRB)}

CRB consists of fuzzy rules with corrected weights obtained after applying MTM. This rule base is stored in file: RULES.DAT with the same form as for CW.DAT (described in the previous section). An example of obtained new rule base after applying the Mobius Transform Module (MTM) for the example described above is the following:

Rule 1:

$$
\begin{aligned}
& \text { Headache } \rightarrow \text { Neurasthenia by Deficiency of Yin Kidney }(0.4) \\
& \text { Headache } \rightarrow \leftarrow \text { Neurasthenia by Deficiency of Yin Kidney }(0.0)
\end{aligned}
$$

Rule 2:

Drowsiness $\rightarrow$ Neurasthenia by Deficiency of Yin Kidney (0.3)

Drowsiness $\rightarrow \leftarrow$ Nearasthenia by Deficiency of Yin Kidney $(0.0)$

Rule 3:

Tinghing in the ears $\rightarrow$ Newrasthenia by Deficiency of Yin Kidney (0.2)

Tinghing in the ears $\rightarrow$ Neurasthenia by Deficiency of Yin Kidney (0.0) Rule 4:

Lumbar Pain $\rightarrow$ Neurasthenia by Deficiency of Yin Kidney $(0.3)$

Lumbar Pain $\rightarrow \leftarrow$ Neurasthenia by Deficiency of Yin Kidney (0.0)

Rule 5:

$\because$ Pulse: tense, small $\rightarrow$ Neurasthenia by Deficiency of Yin Kidney (0.5)

Pulse: tense, small $\rightarrow \leftarrow$ Neurasthenia by Deficiency of Yin Kidney (0.0) Rule 6:

Bitter mouth $\rightarrow$ Neurasthenia.hy Deficiency of Yin Kidney $(0.0)$

Bitter mouth $\rightarrow \leftarrow$ Neurastheniathy Deficiency of Yin Kidney (0.7)

Rule 7: 
Headache $\wedge$ Drowsiness $\wedge$ Tinghing in the ears $\wedge$ Lumbar Pain $\wedge$ Bitter mouth $\rightarrow$ Neurasthenia by Deficiency of Yin Kidney (0.859375)

Headache $\wedge$ Drowsiness $\wedge$ Tinghing in the ears $\wedge$ Lumbar Pain $\wedge$ Bitter mouth $\rightarrow \leftarrow$ Neurasthenia by Deficiency of Yin Kidney (0.000000).

\subsection{VIEW Module (VIEWM)}

This module allows the user to see and verify the content of the conditional weight system B. An example is shown in Figure 5.

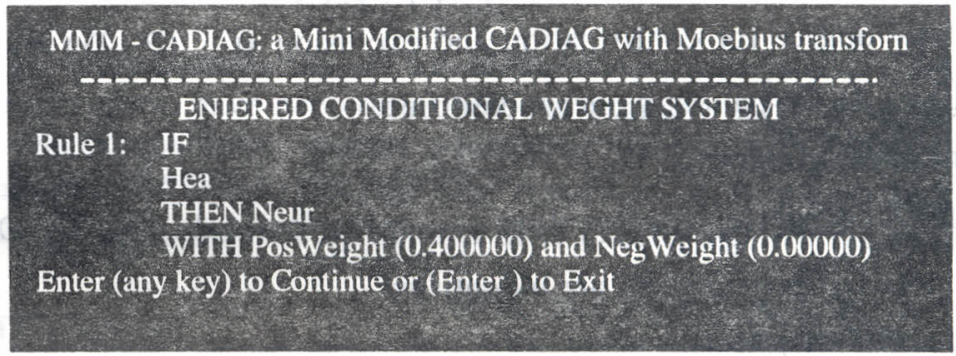

Figure 5: An example of entered CWS

User can consider also the corrected rule base after applying of Mobius transform algorithm. An example is expressed in Figure 6.

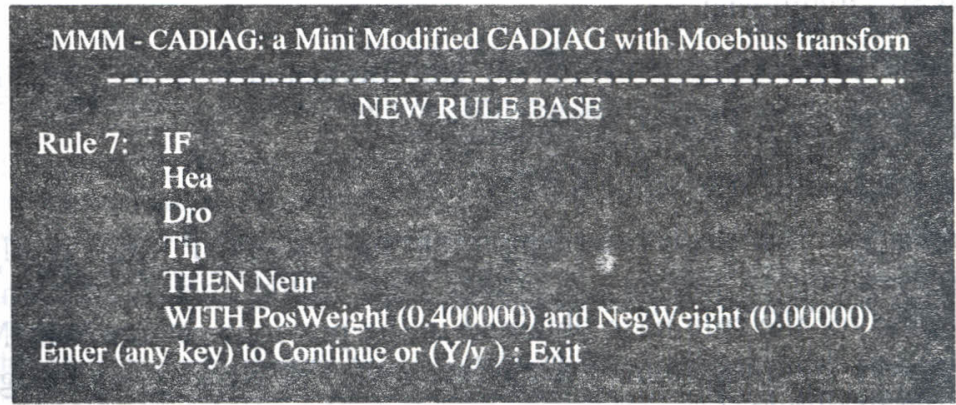

Figure 6: An example of the corrected rule

\subsection{Inference Engine Modile (IEM)}

Using Diagnosis menu of MMiNi CADIAG, the user can receive advices from MMMCADIAG based on input symptoms. Let given a new rule obtained after applying MTM for conditional weight system $\beta$, for example the'new rule in the previous section, the total degree for confirmation and exclusion of diagnosis $D_{j}$ by patient $P_{q}$ from observed symptoms $S_{i}$ is:

$$
\mu_{R_{p+1}^{\text {lot }}}\left(P_{,}, \nu_{j}\right)=\mu_{R_{p+1}^{+}}\left(P_{q}, D_{j}\right) Y \mu_{R_{p+1}^{-}}\left(P_{q}, D_{j}\right)
$$

in $[-1,1]$

where

$$
\begin{aligned}
& \mu_{R_{p+1}^{+}}\left(P_{q}, D_{j}\right)=\operatorname{Max}_{E_{q}}\left[\mu_{R_{S p}^{+}}\left(E_{q}^{\prime}, D_{j}\right)\right] \\
& \mu_{R_{p D}}\left(P_{q}, D_{j}\right)=\operatorname{Max}_{E_{q}^{\prime}}\left[\mu_{R_{S 1}^{-}}\left(E_{q}^{\prime}, D_{j}\right)\right]
\end{aligned}
$$

where $E_{q}^{\prime}$ varies over all elementary conjunctions of symptoms for which $\mu_{R_{S t l}^{\prime}}\left(E_{q}^{\prime}, D_{j}\right)$ or $\mu_{\mathrm{R}_{\mathrm{SI}}}\left(\mathrm{E}_{\mathrm{q}}^{\prime}, \mathrm{D}_{\mathrm{j}}\right)$ is positive. 
Based on the observed symptoms by patient (for example, 'unknown', surely present, surely absent), the Inference of MMM-CADIAG infer the following conclusions (one case for each diagnosis):

- Confirmed diagnosis:

The total degree for confirmation and exclusion $\mu_{\mathrm{R}_{p \mid 1)}^{\mathrm{iot}}}\left(\mathrm{P}_{\mathrm{q}}, \mathrm{D}_{\mathrm{j}}\right)=1.00$, i. e. confirmed diagnoses $\mathrm{D}_{\mathrm{j}}$ for patient $\mathrm{P}_{4}$, are indentified.

- Excluded diagnosis:

The total degree for confirmation and exclusion $\mu_{\mathrm{R}_{\mathrm{P} \mid \mathrm{i}}^{\text {iaf }}}\left(\mathrm{P}_{\mathrm{q}}, \mathrm{D}_{\mathrm{j}}\right)=-1.00$, i. e. disconfirmed diagnoses $\mathrm{D}_{\mathrm{j}}$ for patient $\mathrm{p}_{\mathrm{q}}$, are indentified.

- 'Unknown' diagnosis:

The total degree for confirmation and exclusion $-\varepsilon \leq \mu_{\mathrm{R}_{\mathrm{p} \mid \mathrm{f}}^{\mathrm{tot}}}\left(\mathrm{P}_{\mathrm{q}}, \mathrm{D}_{\mathrm{j}}\right) \leq \varepsilon$, i. e. 'unknown' diagnoses $\mathrm{D}_{\mathrm{j}}$ for patient $\mathrm{P}_{\mathrm{q}}$, are indentified.

- 'Unlikely' diagnosis:

The total degree for confirmation and exclusion $-1 \leq \mu_{R_{p+1}^{\text {iot }}}\left(P_{4}, D_{j}\right) \leq-\varepsilon$, i. e. "unlikely' diagnoses $\mathrm{D}_{\mathrm{j}}$ for patient $\mathrm{P}_{\mathrm{q}}$, are indentified.

- 'Likely' diagnosis:

The total degree for confirmation and exclusion $\varepsilon \leq \mu_{\mathrm{R}_{(+1)}^{\min }}\left(\mathrm{P}_{\mathrm{q}}, \mathrm{D}_{\mathrm{j}}\right) \leq 1$, i. e. 'Likely' diagnoses $\mathrm{D}_{\mathrm{j}}$ for patient $\mathrm{P}_{4}$, are indentified.

(where $\varepsilon$ takes the low value 0.02 )

Assume that the observed symptoms by patient $P_{4}$ are surely present: Headache, Drowsiness, Tinghing in the ears, Lumbar Pain, Bitter mouth (5 symstoms). Applying this Inference Engine for the rule base obtained in the previous section, MMM-CADIAG concludes 'Likely' diagnosis Neurasthenia by Deficiency of Yin Kedney with degree 0.4 for patient $\mathrm{P}_{4}$. One can see the in Figure 7.

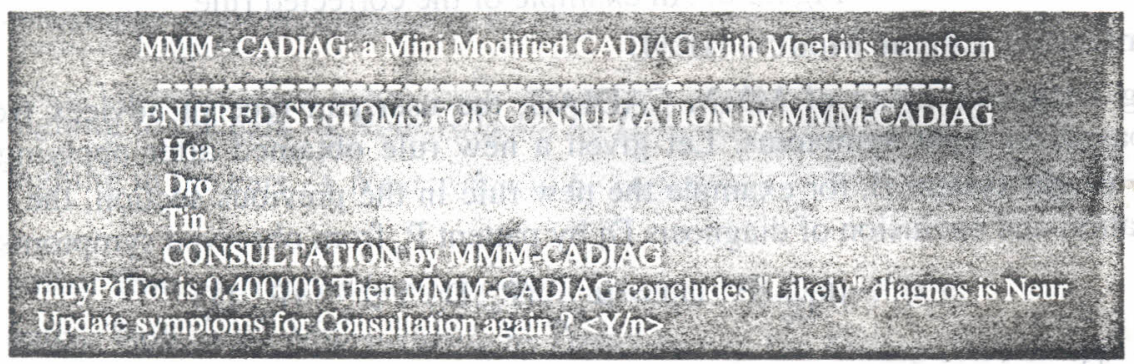

Figure 7: Example of Diagnosis by MMM-CADIAG

\section{IMPLEMENTATION OF PROTOTYPE}

The prototype of the MMM-CADIAG system is experimentally implemented in $\mathrm{C}++$ Programming language on $\mathrm{PC} / \mathrm{IBM}$ computers. In this version, there are some limitations:

- Number of rules is up to 10

- Number of symptoms is up to 10 
- Length of symptom and disease is up to 40 characters. Symmtom and Disease must be written in continuous characters.

- Rules with shorter antecedents earlier.

- Symptom name is not substring of other symptom name.

At present, MMM-CADIAG is under development to be completed.

\section{CONCLUSION}

In this paper, the main components of the MMM-CADIAG system, a Mini Modified CADIAG with Mobius transform have been described. An example expressing how the system works is given. The MMM-CADIAG system guarantees that applying Mobius Transform Module to find new rule base of fuzzy rules, the total degree of confirmation and exclusion of diagnosis $\mathrm{D}_{\mathrm{j}}$ by patient $\mathrm{P}_{q}$ from observed symptoms $\mathrm{Si}$ is exactly egal to the total conditional weight system $\beta$.

\section{ACKNOWLEDGEMENT}

This work was partly supported by the COPERNICUS grant of the European Union "MUM" (Managing Uncertainty in Medicien), No.10053. Thanks due to Prof. P. Hajek for advice and Dr. M. Daniel for fruitful discussions.

\section{REFERENCES}

(1) Adlassnig K. P, Fuzzy Set Theory in Medical Diagnosis. IEEE Transaction on Systems, Man, and Cybernetics, SMC $16(1986) 260-265$.

[2] Buchanan B. G, Shorliffe E. H, Rule-Based expert systems. MYCIN experiments of the Stanford Heuristic Programming Project (Addition - Wesley, Reading, MA, 1984).

[3]. Hajek P. Havranek T, Jirousek R, Uncertain Information Processing in Expert Systems. (CRC PRESS, 1992)

[4] Hajek P. Nguyen Hoang Phuong. Mobius transform for CADIAG-2 Tech. Rep. V-650, ICS, Academy of Sciences of the Czech Republic. (1995) (16p) and in Abst Book of Proc, of 3th Inter. Conf. on Fuzzy Set Theory and its Applications, Jan, 22 26, 1996, Liptovsky Mikulas, Slovak Rep, p. 28. $1653-1658$.

[5] Nguyen Hoang Phuong, Approach to combining negative and positive evidence in CADIAG-2 Proc. EUFIT'95 (1995),

[6]. Zadeh L. A. Fuzzy sets, Information and Control, Vol. 8 (1965) 338 - 353.

68.

[7]. Hajek, P, Valdes, J. J, An analysis of MYCIN - like expert systems. Mathware and Soft Coniputing, No.I (1994), 45 -

[8]. Zimmermann, H, J, Fuz=y Set Tht. y; und Its Applications. Kluwer Academic Publishers, Boston/ Dordrecht/ London, (1991), 399p.

[9] K. P. Adlassnig, A Fuzzy Logical Model of Computer - Assisted Medical Diagnosis, Meth, Inform, Meth, Vol. 19, No. 3 (1980) $141-148$

[10]. K. P. Adlassnig, CADIAG: Approaches to Conputer - Assisted Medical Diagnosis. Computers in Biology and Medicien 15 (1985) $315-335$.

[11] K. P. Adlassing, Update on CADIAG-2: A Fuzzy medic al expert system for general internal medicine. W. H. Janko et al. (eds), Progyess in Fuzzy Sets and Systems (1990) ' 1 - 6.

[12]. K. P. Adlassing et al, Approach tr a hospital-based application of a medical expert system, Meth, Inform, Vol. 11. No. 3 (1996) $205-223$.

[13] G. J. Klir, T. A. Folger. Fuzzy sets, Uncertainty, and Information (Prentice - Hall International Editions, 1988).

[14]. Nguyen Hoang Phuong. Fuzzy Set Theory and Medical Expert Systems. Survey and Model. Proc. SOFSEM'95: Theory and Practice in Informatics, Lecture Notes in Computer Science, No. 1012, Springer - Verlag (1995), 431 - 436.

[15]. P. Hajek, Nguyen Hoang Phuong CADIAG-2 and MYCIN - like SYSTEMS. Submitted for J. Artificial Intelligence in Medicien. Vol. 9, 1997, 241-259.

[16] Nguyen Hoang Phuong Some Contrihutions to Fuzzy Expert Systemss with emphasis on Medicine. Ph. D. Thesis. Technical University of Vienna (1995). 